\title{
Trajectory Optimization for Walking Robots with Series Elastic Actuators
}

\author{
Alexander Werner, Roberto Lampariello and Christian Ott
}

\begin{abstract}
Series elastic actuators are a promising feature for future walking robots. Utilizing the natural oscillation of the elastic actuation can lead to efficient locomotion. However finding a matching combination of joint stiffness and actuator input is challenging as these robots share the properties of underactuated and hybrid dynamic systems. In this paper an optimization problem for cyclic walking of a robot with series elastic actuators is formulated and a flatness based solution method is presented. The method efficiently generates trajectories which exploit the actuator capabilities while respecting all limitations of the system. Additionally the relevant aspects of the trajectories for the design of a flexible robot are discussed.
\end{abstract}

\section{INTRODUCTION}

Generation of walking and running trajectories for humanoid robots has been an active research topic in the recent years. This is a challenging field for any planning method as it requires to find feasible trajectories for systems which combine non-linear system dynamics, under-actuation and hybrid dynamics.

The main purpose of off-line optimization is to provide an on-line controller with a feed-forward term for input of the system and a desired system state. This is especially useful in the context of tight system limits and systems for which only control approaches are viable with simplify the system behavior drastically. Even though computing capacities of robotic systems improve, the existing methods can not handle instantaneous computation of optimal trajectories which take the full system dynamics and constraints into account. In addition to the application in trajectory generation the methods can also be used in the design phase of such a system. This can provide valuable insight into the usefulness of various stiffness settings.

For electrically actuated robots with mechanical gears series elastic actuators provide better performance and mechanical robustness in comparison to a rigid robot. Also torque control can be easily implemented. High performance and efficiency are provided by the exploitation of the natural system dynamics, which is especially possible for cyclic tasks. Robustness is ensured by the decoupling of the gears from high link side torques. This is done without sacrificing the versatility of the robot like robots using parallel springs do.

*All authors are with the Institute of Robotics and Mechatronics (DLR). E-mail: firstname.lastname@dlr.de
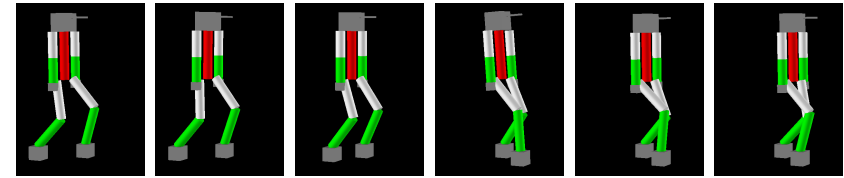

Fig. 1. Snapshots from an energy optimal motion taken $0.11 \mathrm{~s}$ apart.

However the choice of actuators and stiffness settings as well as control inputs for a given task which makes use of the elasticities, remains challenging. The main contribution of this paper is to provide an efficient way to find an energy optimal trajectory which profits from the capabilities of the series elastic actuators. The method presented herein uses a compact description of the possible trajectories which minimize the number of parameters in the optimization problem.

In our previous work [1] we describe an optimizationbased method to generate cyclic walking trajectories for a three-dimensional rigid-joint walking robot. In this work, which builds on the previous paper, we extend the concept to flexible robots. The presented method allows to efficiently parameterize the full state through one spline trajectory using the flatness properties of the system. In addition the problem formulation now contains a double support phase and addresses the distribution of the forces on the contact point explicitly. As the proposed method uses inverse dynamics, this includes solving the problem of distributing the contact forces on two flat feet. The implementation presented in [1] was improved by using analytic Jacobian matrices for the constraints and the cost-function.

The experimental validation of the trajectories described in [1] led to the conclusion that even with a robot which is designed to be rigid, taking into account the inherent flexibility and the motor-side inertias is relevant for the successful execution of trajectories.

The previous work presented energy-optimal trajectories for our three-dimensional, 12 degree of freedom walking machine DLR Biped [2]. The approach presented herein extended this to flexible joints, albeit for the planar case.

Earlier research focused on rigid-body robots [3], but since then, several elastic systems using legged locomotion have been developed [4]-[6].

For stiff humanoid robots the 3D case has been extensively researched: [3], [7].

The introduction of flexibility in robotic systems for the trajectory planning task was addressed in [8], to 


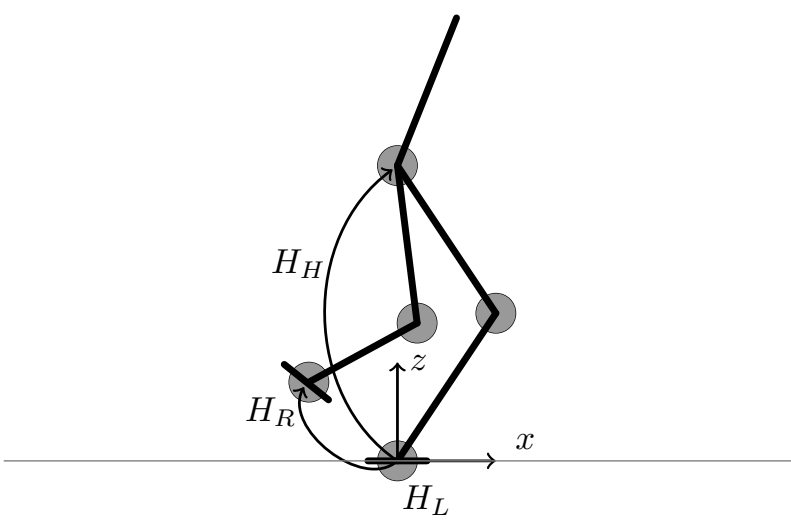

Fig. 2. Kinematics of the model

treat flexibility in the links, however to generate motion of a manipulator arm along a specified path. The more general problem, for which no reference path is provided, was tackled in [9] and in the paper cited therein, for humanoid systems with flexible elements placed in parallel to passive or to actuated joints. The method of solution in [9] is based on the direct multiple shooting approach.

[10] motivates nicely the use of optimization for design and trajectory generation in the context of series elastic humanoids, also using biarticulation.

\section{Problem statement}

The problem formulated here leads to trajectories repetitive, cyclic trajectories which are energy optimal and within the system limits. This section proceeds with the used model and a description of the gait pattern.

\section{A. Humanoid walking model with elastic joints}

This study uses a minimal model for a planar walking robot with two feet $\left(N_{C}=2\right)$ shown in Fig. 2, The planar model has $N_{B}=3$ non-actuated degrees of freedom $\boldsymbol{x}$ for the position and orientation of the base body, two translational DOF along $x$ and $z$ and one rotational DOF around $y$. The robot consists of $N_{J}=6$ actuated joints $\boldsymbol{q}$, three per leg. The approach described herein is fully independent of the kinematics of the robot and can also be used for the three-dimensional case.

The configuration space of the complete model combines base $\left(N_{B}\right)$ and joint space coordinates $\left(N_{J}\right)$ and is given as $\boldsymbol{y}$ with the dimension $N_{D}=N_{B}+N_{J}$ :

$$
\boldsymbol{y}=\left[\begin{array}{ll}
\boldsymbol{x} & \boldsymbol{q}
\end{array}\right]^{T} \quad \boldsymbol{q}=\boldsymbol{S} \cdot \boldsymbol{y}
$$

with $\boldsymbol{S}=\left[\begin{array}{ll}\mathbf{0} & \boldsymbol{I}\end{array}\right] \in \mathbb{R}^{N_{J} \times N_{D}}$ the projector for the actuated states defined by $\boldsymbol{q}$.

The stance foot, denoted by index L, is defined to be located in the origin, thus the swing foot position, denoted by $\boldsymbol{H}_{R}$, defined by the forward kinematics is only a function of $\boldsymbol{q}$ :

$$
\begin{aligned}
\boldsymbol{H}_{L} & =\boldsymbol{I} \\
\boldsymbol{H}_{R}(\boldsymbol{q}) & =f_{H R}(\boldsymbol{q}) \\
\boldsymbol{J}_{R}(\boldsymbol{q}) & =f_{J R}(\boldsymbol{q})
\end{aligned}
$$

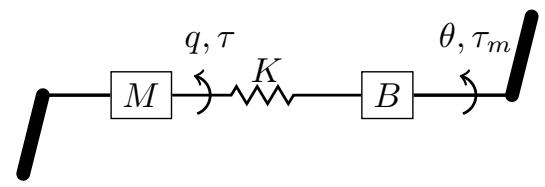

Fig. 3. The series elastic actuator attached to two rigid links. The model contains the link side inertia $M$, stiffness of the elastic element $K$ and motor side inertia $B$

where $\boldsymbol{H}_{R} \in \mathbb{R}^{3 x 4}$ is a homogeneous transformation matrix that defines the position and orientation of the right foot with respect to the inertial frame and $\boldsymbol{J}_{R}$ the associated body Jacobian. This assumptions requires that the contact between the stance foot and the floor is fixed.

This description uses free-floating version of the equations of motion to describe the force-distribution problem in a more consistent way. The dynamic equations of the system are given by

$$
\boldsymbol{M}(\boldsymbol{y}) \ddot{\boldsymbol{y}}+\boldsymbol{C}(\boldsymbol{y}, \dot{\boldsymbol{y}}) \dot{\boldsymbol{y}}+\boldsymbol{g}(\boldsymbol{y})=\boldsymbol{S}^{T} \boldsymbol{\tau}+\sum_{i=0}^{N_{C}} \boldsymbol{J}_{i}^{T}(\boldsymbol{y}) \boldsymbol{W}_{i}
$$

with $\boldsymbol{M} \in \mathbb{R}^{N_{D} \times N_{D}}$ as inertia matrix, $\boldsymbol{C} \in \mathbb{R}^{N_{D} \times N_{D}}$ as centrifugal matrix, $\boldsymbol{g} \in \mathbb{R}^{N_{D}}$ as gravity terms, $\boldsymbol{\tau} \in \mathbb{R}^{N_{J}}$ as actuator torques, $\boldsymbol{J}_{i} \in \mathbb{R}^{6 \times N_{D}}$ as contact Jacobian and $\boldsymbol{W}_{i} \in \mathbb{R}^{6}$ as reaction wrench. In addition to this rigid body model the series elastic joints are now coupled through the potential coupling through the elastic element as shown in Fig. 3 forming a reduced elastic joint model [11]:

$$
\begin{aligned}
\tau & =\boldsymbol{K}(\boldsymbol{\theta}-\boldsymbol{q}) \\
\boldsymbol{B} \cdot \ddot{\boldsymbol{\theta}}+\boldsymbol{\tau} & =\tau_{m}
\end{aligned}
$$

where $\boldsymbol{\theta} \in \mathbb{R}^{N_{J}}$ are the motor side positions, $\boldsymbol{K} \in$ $\mathbb{R}^{N_{J} \times N_{J}}$ is the diagonal matrix of the stiffnesses for the elastic elements, $\boldsymbol{B} \in \mathbb{R}^{N_{J} \times N_{J}}$ is the diagonal matrix of the effective inertias of the motors and gears on the link side and $\boldsymbol{\tau}_{m} \in \mathbb{R}^{N_{J}}$ the motor torques. $\boldsymbol{K}$ is assumed to be constant.

\section{B. Gait description}

The gait pattern of interest is composed of a single support phase (SSP) and a double support phase (DSP). The cyclic gait patterns relevant for continuous gait can be described with the parameters stride length $s_{x}$ and cycle time $t_{f}$.

As cyclic walking is composed of two repeated gait patterns which are similar to each other, only one step is described and optimized. In the described gait the feet maintain surface contact on a level ground.

As the task is to find cyclic walking gait patterns, the trajectories must satisfy the continuity requirements for start and end configuration of the robot:

$$
\begin{aligned}
& \boldsymbol{q}\left(t_{f}\right)=\boldsymbol{F} \cdot \boldsymbol{q}\left(t_{0}\right) \\
& \boldsymbol{\theta}\left(t_{f}\right)=\boldsymbol{F} \cdot \boldsymbol{\theta}\left(t_{0}\right)
\end{aligned}
$$


where $\boldsymbol{F}$ exchanges the states of the right and left leg joints. Additionally the derivatives of (8) and (9) have to be fulfilled to guarantee that all states are continuous.

\section{Optimization Problem}

In this section the optimization problem is described which implements the previously described trajectory planning task. The formulation is similar to [1] and is here extended to find an optimal trajectory for the case of a robot with series elastic actuators. The abstract optimization problem is defined as:

$$
\begin{array}{cc}
\underset{\boldsymbol{p}}{\operatorname{minimize}} & \Gamma(\boldsymbol{p}) \\
\text { subject to } & \boldsymbol{e}(\boldsymbol{p})=0 \\
& \boldsymbol{h}(\boldsymbol{p})<0
\end{array}
$$

with the optimization parameters $\boldsymbol{p} \in \mathbb{R}^{N_{P}}$, the cost functional $\Gamma \in \mathbb{R}(\boldsymbol{p})$, the equality constraints $\boldsymbol{g}(\boldsymbol{p})$ and the inequality constraints $\boldsymbol{h}(\boldsymbol{p})$.

\section{A. Cost Function}

The chosen cost-function approximates the electric energy consumed by the motor with an non-backdrivable harmonic drive gear:

$$
\begin{aligned}
\Gamma(\boldsymbol{p}) & =\int_{t_{0}}^{t_{f}} \sum_{i=0}^{N_{J}} f\left(\dot{\theta}_{i} \cdot \tau_{m, i}\right) \mathrm{d} t \\
f(x) & = \begin{cases}x & \text { if } x \geq 0 \\
0 & \text { if } x<0\end{cases}
\end{aligned}
$$

\section{B. Equality Constraints}

The equality constraints are used to ensure

$$
\left.\boldsymbol{H}_{R}(t)=\boldsymbol{H}_{\text {goal }} \quad \forall t \in\right] t_{s w} ; t_{f}[
$$

where $\boldsymbol{H}_{\text {goal }}$ is the position of the swing foot at the end of the step. Additionally the velocity of the swing foot $\boldsymbol{V}_{R}$ at the time of contact $t_{s w}$ set to be zero, so that no energy is removed from the system:

$$
\boldsymbol{V}_{R}\left(t_{s w}\right)=\mathbf{0}
$$

In order to have a continuous transition of all states to the follow step, all differential states are constraint at $t_{f}$ :

$$
\frac{\partial^{n}}{\partial t^{n}} \boldsymbol{V}_{R}\left(t_{f}\right)=\mathbf{0} \quad \forall n \in[0 ; 2] .
$$

These Cartesian position and velocity constraints in the planar case are implemented with 3 equations each.

\section{Inequality Constraints}

The inequality constraints described below are required to ensure that the robot can be assumed to be fixed base. This is required to be able to describe the complete configuration of the robot $\boldsymbol{y}$ through the actuated joints $\boldsymbol{q}$. The constraints ensure a stable surface contact of the left foot in the single support phase and of both feet during the double support phase. These constraints remain unchanged with respect to the rigidbody problem described in [1].

Support Area is implemented through constraining the center of pressure given for the feet in contact:

$$
-\frac{s_{w}}{2}<\frac{\tau_{L, y}}{F_{L, z}}<\frac{s_{w}}{2}
$$

where $s_{w}$ is the length of the foot.

Friction Cone limits the tangential forces of the feet in contact. It also constrains the contact force to fulfill the unilaterality condition.

$$
\left|F_{L, x}\right|<\mu F_{L, z}
$$

where $F_{L, x}$ is the contact force in $\mathrm{x}$ direction and $\mu$ is the friction coefficient.

Additional constraints are required to guarantee the feasibility of the motion with respect to actuator and kinematic limits. It is important to realize that in contrast to the rigid-body problem, the torque and velocity constraints are now on the actuator side.

Joint position

$$
q_{i, \min }<q_{i}(t)<q_{i, \max } \quad \forall i \in\left[0 ; N_{J}\right]
$$

Actuator velocity is given either by the characteristics of the motor itself and by the limitations of the gears:

$$
\left|\dot{\theta}_{i}(t)\right|<\dot{\theta}_{i, \max } \quad \forall i \in\left[0 ; N_{J}\right]
$$

Actuator torque, like actuator velocity is a limit imposed by the actual components:

$$
\left|\tau_{m, i}(t)\right|<\tau_{m, i, \max } \quad \forall i \in\left[0 ; N_{J}\right]
$$

Spring deflection is limited because most interesting spring types have a geometric limitation. This can also be seen as a limit on the maximum of energy stored in the system.

$$
\left|\theta_{i}(t)-q_{i}(t)\right|<\phi_{i, \max } \quad \forall i \in\left[0 ; N_{J}\right]
$$

Collision avoidance is computed from the minimal distance between the floor and the swing foot which is approximated by a set of points $\boldsymbol{x}_{i}$ given in the local coordinate system of the foot. The minimal distance of all these points to the floor is then computed.

$$
\begin{array}{r}
\tilde{\boldsymbol{x}}_{i}(t)=\boldsymbol{H}_{R}(t) \cdot \boldsymbol{x}_{i} \\
\min \left\{\tilde{z}_{i}(t)\right\}>0
\end{array}
$$

\section{Solution Method}

To solve the described optimization problem, nonlinear programming is applied. This is described in detail in section IV-A. In contrast to a shooting-based method the state is parameterized and the required actuator torques and reaction forces are computed by inverse dynamics as described in the sections IV-B and IV-C.

The cost- and constraint-functions are discretized in time at $N_{v}$ equidistant points:

$$
t_{0} \leq t_{i} \leq t_{f} \quad \forall i \in\left[0 ; N_{v}\right]
$$


The inverse dynamics algorithm for this fixed base system avoids solving non-linear differential equations through numerical integration, which improve optimization speed. The vector of optimization parameter is composed of two parts

$$
\boldsymbol{p}=\left[\begin{array}{ll}
\boldsymbol{p}_{S} & \boldsymbol{p}_{\tau}
\end{array}\right]
$$

which are described in the following sections.

\section{A. Parameterization}

The position of the robot joints, denoted by $\boldsymbol{q}$ us defined by the function $\boldsymbol{q}=f\left(\boldsymbol{p}_{S}, t\right)$ with $\boldsymbol{p}_{S}$ a subset of the optimization parameters. $\boldsymbol{f}\left(\boldsymbol{p}_{S}, t\right)$ is a fifth order basic spline which is linear in the optimization parameters $\boldsymbol{p}_{S}$ and guarantees continuous derivatives up to $\dddot{\boldsymbol{q}}$.

As shown in the following section, he motor side states $\boldsymbol{\theta}$ and $\dot{\boldsymbol{\theta}}$ can be determined through the derivatives $\ddot{\boldsymbol{q}}$ and $\dddot{\boldsymbol{q}}$ using the flatness properties of the system.

Using the model assumption that the stance foot maintains a stable contact and the position of the joints $\boldsymbol{q}$, the base states $\boldsymbol{x}$ can be computed by solving (2) so that the full system state $\boldsymbol{y}$ is know. This is repeated for higher derivatives to obtain $\dot{\boldsymbol{y}}, \ddot{\boldsymbol{y}}$ and $\dddot{\boldsymbol{y}}$.

Continuity of the system state $\boldsymbol{y}$ within the phases of the trajectory is guaranteed by the spline properties. There are two phase boundaries where the contacts with the environment switch: from the single support to the double support phase and from the double support phase to the single support phase, considering (8) and (9).

On these boundaries, continuity for the link side states $\boldsymbol{y}$ and $\dot{\boldsymbol{y}}$ can be ensured by applying the spline boundary conditions explained below.

The spline boundary conditions can be formulated as a number of constraints on the spline parameters set $\boldsymbol{p}_{S}$ :

$$
\frac{\partial^{n}}{\partial t^{n}} \frac{\partial}{\partial \boldsymbol{p}_{S}} f_{\text {spline }}\left(\boldsymbol{p}_{S}, t_{0}\right) \stackrel{!}{=} \frac{\partial^{n}}{\partial t^{n}} \frac{\partial}{\partial \boldsymbol{p}_{S}} f_{\text {spline }}\left(\boldsymbol{p}_{S}, t_{f}\right)
$$

for $n \in[0 ; 4]$ to guarantee the required degree of continuity. As basic splines are linear in the parameters, the remaining null space $\hat{\boldsymbol{p}}_{S}$ of these constraints can be used for optimization. It is beneficial to use a non-uniform knot point distribution with multiple knots at the trajectory boundaries to avoid restricting the flexibility of the spline at the boundaries.

Continuity is also required for the motor side states $\boldsymbol{\theta}$ and $\dot{\boldsymbol{\theta}}$ or equivalently for $\ddot{\boldsymbol{y}}$ and $\dddot{\boldsymbol{y}}$. These states depend on $\boldsymbol{y} \ldots \dddot{\boldsymbol{y}}$ and on the external forces $\boldsymbol{W}$. Section IV-C describes how continuity is maintained for the external forces.

\section{B. Inverse Dynamics: Single Support}

This method parameterizes the space of all solutions through the previously described splines, which define all joint states, and through (2) also the complete system state.

For this it is initially assumed that any contact force is feasible. The constraints on the contact force such as maximum tangential forces and unilaterality are enforced through constraints in the optimization problem.

Given the state $\boldsymbol{y}, \dot{\boldsymbol{y}}$ and $\ddot{\boldsymbol{y}}$ the link-side torques $\boldsymbol{\tau}$ and the contact wrenches $\boldsymbol{W}_{i}$ can be computed using (5).

Differentiating (5) in time results in the following two expressions:

$$
\begin{aligned}
\dot{\boldsymbol{O}}(\boldsymbol{y}, \dot{\boldsymbol{y}}, \ddot{\boldsymbol{y}}, \dddot{\boldsymbol{y}}):= & \dot{\boldsymbol{M}} \ddot{\boldsymbol{y}}+\boldsymbol{M} \dddot{\boldsymbol{y}}+\dot{\boldsymbol{C}} \dot{\boldsymbol{y}}+\boldsymbol{C} \ddot{\boldsymbol{y}}+\dot{\boldsymbol{g}}=(26) \\
= & \boldsymbol{S}^{T} \dot{\boldsymbol{\tau}}+\sum_{i=0}^{N_{C}}\left[\dot{\boldsymbol{J}}_{i}^{T} \boldsymbol{W}_{i}+\boldsymbol{J}_{i}^{T} \dot{\boldsymbol{W}}_{i}\right] \\
\ddot{\boldsymbol{O}}(\boldsymbol{y}, \dot{\boldsymbol{y}}, \ddot{\boldsymbol{y}}, \dddot{\boldsymbol{y}}, \dddot{\boldsymbol{y}})= & \boldsymbol{S}^{T} \ddot{\boldsymbol{\tau}}+ \\
& +\sum_{i=0}^{N_{C}}\left[\ddot{\boldsymbol{J}}_{i}^{T} \boldsymbol{W}_{i}+2 \dot{\boldsymbol{J}}_{i}^{T} \dot{\boldsymbol{W}}_{i}+\boldsymbol{J}_{i}^{T} \ddot{\boldsymbol{W}}_{i}\right]
\end{aligned}
$$

where the function dependencies have been removed for clarity. Now given $\boldsymbol{\tau}$ and $\dot{\tau}$ from 26 , the motor side states $\boldsymbol{\theta}$ and $\dot{\boldsymbol{\theta}}$ can be computed using (6).

Through solving (7) for $\ddot{\boldsymbol{\theta}}$ and substituting it into (6), differentiated twice, yield:

$$
\ddot{\boldsymbol{\tau}}=\boldsymbol{K}(\ddot{\boldsymbol{\theta}}-\ddot{\boldsymbol{q}})=\boldsymbol{K}\left(\boldsymbol{B}^{-1}\left(\boldsymbol{\tau}_{m}-\boldsymbol{\tau}\right)-\ddot{\boldsymbol{q}}\right)
$$

which allows to compute the actuator torques $\boldsymbol{\tau}_{m}$.

To improve performance the results of the equations (5), (26) and (27) can be computed through a recursive algorithm like the articulated-body algorithm.

\section{Inverse Dynamics: Double Support}

Equations (5),26) and (27) are redundant for the case of multiple contact points as it is the case for the double support phase. To control the distribution of forces through the optimization problem, the equations are modified to contain the diagonal weight matrix $\boldsymbol{\alpha}\left(\boldsymbol{p}_{\tau}, t\right) \in \mathbb{R}^{6 \times 6}$. The elements of the weight matrix are controlled by additional optimization parameters $\boldsymbol{p}_{\tau}$. The modified equations are:

$$
\begin{aligned}
\boldsymbol{O}(\boldsymbol{y}, \dot{\boldsymbol{y}}, \ddot{\boldsymbol{y}}, \dddot{\boldsymbol{y}}) & =\boldsymbol{S}^{T} \boldsymbol{\tau}+\left[\begin{array}{ll}
\boldsymbol{J}_{1}^{T} & \boldsymbol{J}_{2}^{T}
\end{array}\right]\left[\begin{array}{l}
\hat{\boldsymbol{W}}_{1} \\
\hat{\boldsymbol{W}}_{2}
\end{array}\right] \\
\hat{\boldsymbol{W}}_{1} & =\boldsymbol{\alpha}\left(\boldsymbol{p}_{\tau}, t\right) \cdot \boldsymbol{W}_{1} \\
\hat{\boldsymbol{W}}_{2} & =\left(\boldsymbol{I}-\boldsymbol{\alpha}\left(\boldsymbol{p}_{\tau}, t\right)\right) \cdot \boldsymbol{W}_{2} \\
\boldsymbol{\alpha}(t) & =f\left(\boldsymbol{p}_{\tau}, t\right)
\end{aligned}
$$

As denoted, the weight matrix is a function of time. Similar to the parameterization of the joint space, a basic spline is used to compute $\boldsymbol{\alpha}\left(\boldsymbol{p}_{\boldsymbol{\tau}}, t\right)$. To enforce that the contact forces are continuous, as mentioned in the last section, boundary conditions are enforced on $\boldsymbol{\alpha}\left(\boldsymbol{p}_{\tau}, t\right)$ :

$$
\begin{aligned}
\boldsymbol{\alpha}\left(\boldsymbol{p}_{\tau}, t_{s w}\right) & =1 \\
\boldsymbol{\alpha}\left(\boldsymbol{p}_{\tau}, t_{f}\right) & =0 \\
\dot{\boldsymbol{\alpha}}\left(\boldsymbol{p}_{\tau}, t\right) & =0 \quad \forall t \in\left\{t_{s w}, t_{f}\right\}
\end{aligned}
$$




\section{Implementation}

Often numeric differentiation is applied to obtain the derivatives in the optimization parameters of the cost function and the constraints required for sequentialquadratic programming. However this is likely to slow convergence. In our implementation the first derivatives are all computed analytically. As implementing the derivatives of the equations of motion or the inverse dynamics with force distribution is very time consuming and error-prone when done exactly, we have chosen to use automatic differentiation in nearly all cases. The Hessian is approximated by the L-BFGS algorithm in the solver as its approximation is less problematic for the convergence.

The interior-point algorithm implemented in IpOpt [12] was used as optimization solver in conjunction with the quadratic solver MUMPS [13]. The robotics optimization library roboptim [14] was used to implement the method.

\section{Results}

For kinematics and dynamics human data was used. The actuator properties used are inertia of $0.7 \mathrm{~kg} \cdot \mathrm{m}^{2}$, maximum torque of $270 \mathrm{Nm}$ and maximum velocity of $5.2 \mathrm{rad} / s$. The elastic element stiffness was chosen to $1000 \mathrm{Nm} / \mathrm{rad}$. The knee angle with constraint to be at least $0.15 \mathrm{rad}$

The step duration was set to $0.7 s$ with a $0.2 s$ double support phase and the stride length to $0.8 \mathrm{~m}$.

\section{A. Optimization}

In the optimization process we used 10 parameters for the single support phase and 8 parameters for the double support phase per joint. This is reduced by the continuity conditions to 8 free parameters per joint. Each element of the diagonal weight matrix $\boldsymbol{\alpha}$ is parameterized by a spline with 2 free parameters. The complete optimization problem has 54 parameters. The cost and constraint functions are sampled at 30 viapoints. Not to violate the Linear Independence Constraint Qualification (LICQ) the equality constraints 12 are active only for a subset of these points.

We use a number of initial guesses generated by a Rapidly-exploring Random Tree (RRT) based algorithm which solely considers the kinematic constraints. Starting from this, we solve an equivalent rigid-body optimization problem where the actuator constraints (18) and (19) are applied on the link side. This solution is then used to start the optimization for the described optimization problem for a robot with series elastic actuators.

Nearly every starting point chosen returns a feasible result. However the results of our global search show that local minima exist in the problem formulation.

\section{B. Trajectories}

The most energy-optimal trajectory found in a global search for the described task is shown in Fig. 4, where

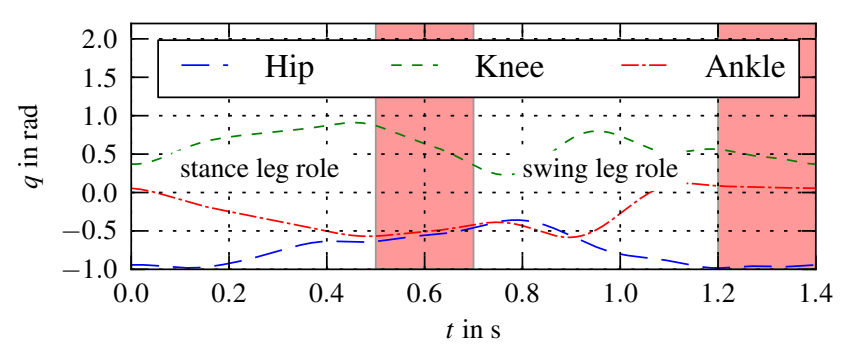

Fig. 4. Energy-optimal joint position trajectories given for $K=$ $1000 \mathrm{Nm} / \mathrm{rad}$.
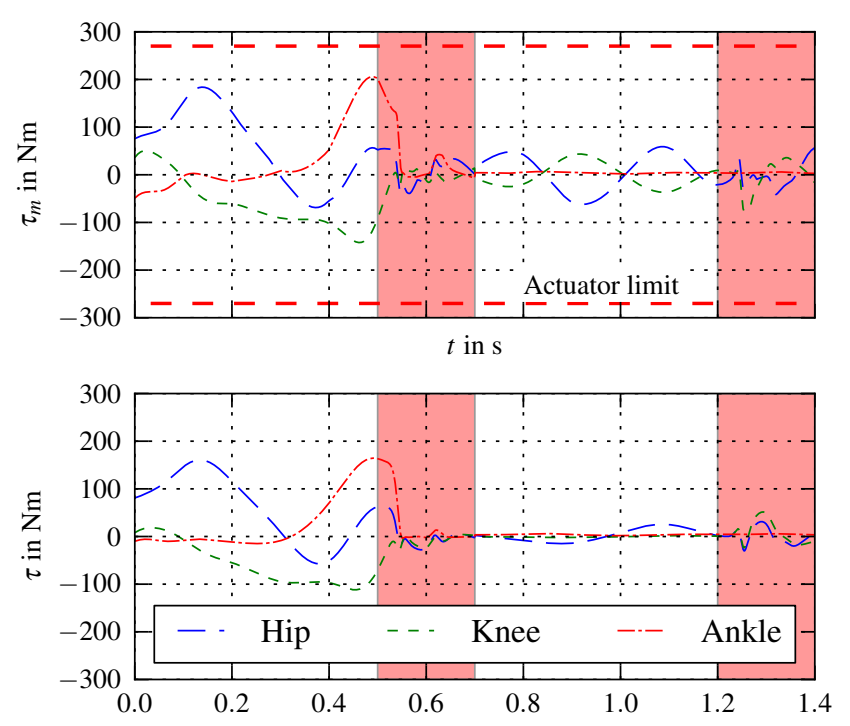

Fig. 5. Top: actuator torque. Bottom: Link-side torque for an energy optimal trajectory for $K=1000 \mathrm{Nm} / \mathrm{rad}$.

the position of the joints is presented. All figures which show the variation of quantities part of the state over time display two consecutive steps. Link and motor side torque trajectories are shown in Fig. 5. As can be seen in there, the actuator torque limits are never touched, which is most likely due to the conservative specification of the task. The found trajectory generates the ground reaction forces shown in Fig. 6 .

To conclude if the elastic elements are used for energy storage and produce significant portions of the trajectory, it is useful to compute the stored energy on a per joint basis, this is shown in Fig. 7. The most simple case of minimal energy consumption is a trajectory where the motor does not move at all and the natural dynamics of the inertia and the spring generate the motion. For single joints these trajectories would resemble an increasing line in a torque-position graph. However, Fig. 8 shows that this is not the case.

\section{Conclusions}

We formulate an optimization problem to find cyclic walking trajectories for a robot with series elastic actuators. The problem includes the single and the double support phase. All necessary limits of a real system 


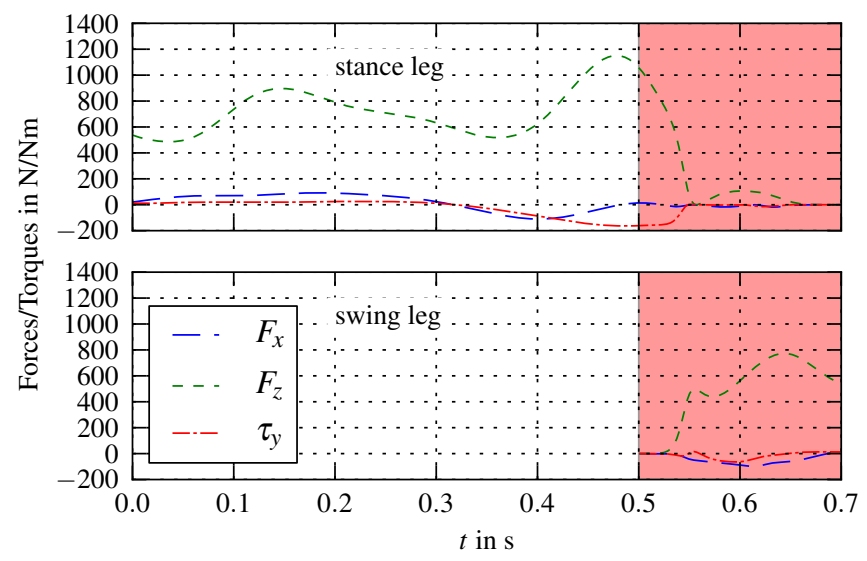

Fig. 6. Ground reaction forces for an energy optimal trajectory for $K=1000 \mathrm{Nm} / \mathrm{rad}$.
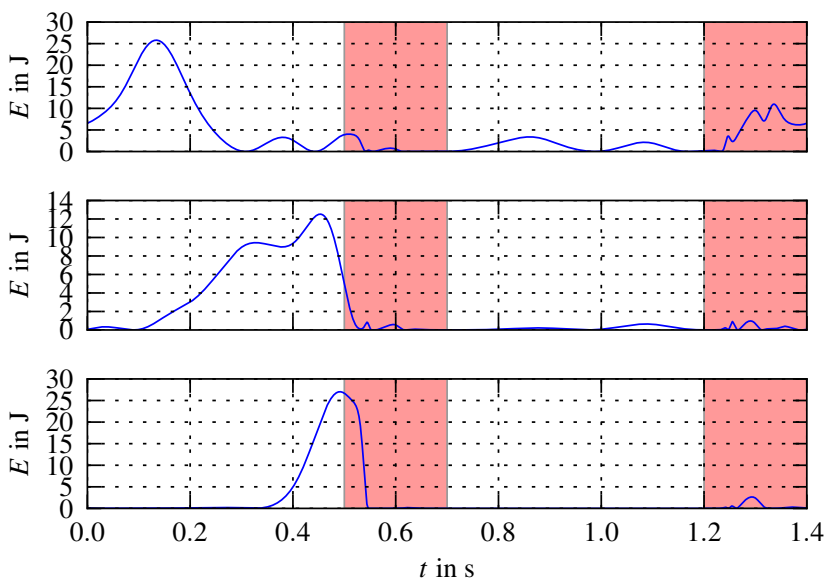

Fig. 7. Energy of leg joints stored in the elastic elements during trajectories given for $K=1000 \mathrm{Nm} / \mathrm{rad}$. From top to bottom: Hip, Knee and ankle Joint.
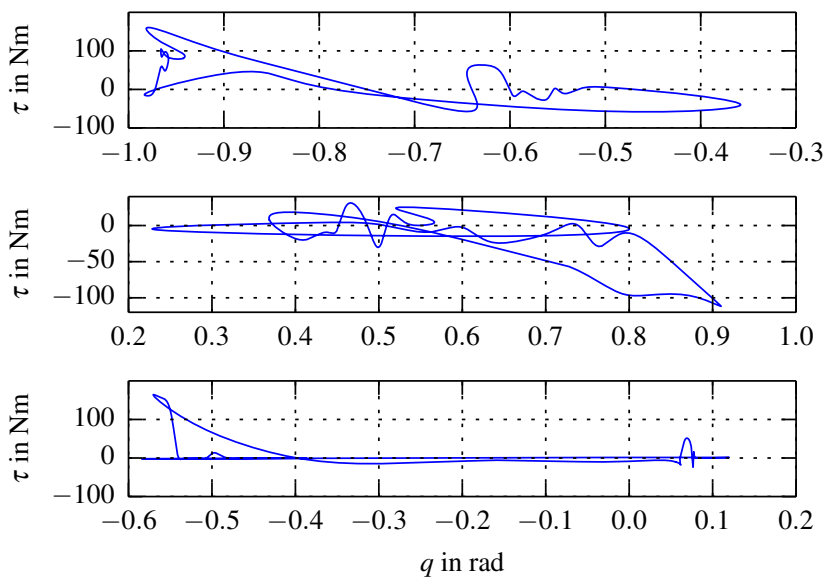

Fig. 8. Link-side torque $\tau$ versus link-side position $q$. Top: Ankle Joint. Middle: Knee Joint. Bottom: Hip Joint. are modelled in the constraints. This includes actuator constraints, constraints inferred by the gears, by the kinematics and by the model assumptions. The model assumption make the model fixed-base which allows us to use the flatness properties of system to parameterize the solution space through the system state $\boldsymbol{y}$ and the derivatives rather then the input $\boldsymbol{\tau}_{m}$.

The applied solution method uses non-linear programming with basic splines to transform the infinite dimensional problem into a finite one. The cost and constraint functions are approximated at a discrete set of points during the trajectory. This allows the optimization to proceed without solving the differential equations of the system dynamics in time. As the inverse system dynamics are not bijective for the distribution of the contact forces for multiple contact points, the force distribution is added to the optimization parameters.

The optimization results show that it is possible to observe if the characteristics of the elastic joints match the task. This way the correct setting for the joint stiffness can be studied.

\section{A. Future Work}

The next step is to match the natural dynamics of the system with the time-constants of the task. This can be achieved by adding either phase times or stiffness settings as optimization parameters. Optimizing mass distribution, on the other hand, is better done using heuristics like moving all components as close to the trunk as possible.

From the results it is evident that stance and swing leg would profit from a different stiffness setting. This could be realized through variable stiffness actuators. In this case the stiffness setting can be parameterized using splines, similar to the trajectory itself.

\section{REFERENCES}

[1] A. Werner, R. Lampariello, and C. Ott, "Optimization-based generation and experimental validation of optimal walking trajectories for biped robots," in Intelligent Robots and Systems (IROS), 2012 IEEE/RSJ International Conference on, 2012, pp. 4373-4379. 1 , 3

[2] C. Ott, C. Baumgärtner, J. Mayr, M. Fuchs, R. Burger, D. Lee, O. Eiberger, A. Albu-Schäffer, M. Grebenstein, and G. Hirzinger, "Development of a biped robot with torque controlled joints," in Humanoid Robots (Humanoids), 10th IEEE-RAS International Conference on, 2010. 1

[3] J. Denk and G. Schmidt, "Synthesis of walking primitive databases for biped robots in 3d-environments," in International Conference on Robotics and Automation, ICRA2003, Proceedings of the IEEE, 2003. 1

[4] J. Grizzle, J. Hurst, B. Morris, H.-W. Park, and K. Sreenath, "Mabel, a new robotic bipedal walker and runner," in American Control Conference, 2009. ACC'09. IEEE, 2009, pp. 2030-2036. 1

[5] F. L. Moro, N. G. Tsagarakis, and D. G. Caldwell, "A humanlike walking for the compliant humanoid coman based on com trajectory reconstruction from kinematic motion primitives," in Humanoid Robots (Humanoids), 2011 11th IEEE-RAS International Conference on. IEEE, 2011, pp. 364-370. 1 .

[6] J. Pratt and B. Krupp, "Design of a bipedal walking robot." in SPIE Defense and Security Symposium, 2008, pp. $69621 \mathrm{~F}-$ $69621 \mathrm{~F}$. 1 
[7] D. Djoudi, C. Chevallereau, and Y. Aoustin, "Optimal reference motions for walking of a biped robot," in Proceedings of the 2005 IEEE International Conference on Robotics and Automation, 2005. 1

[8] A. Mohri, P. K. Sarkar, and M. Yamamoto, "An efficient motion planning of flexible manipulator along specified path," in Robotics and Automation, 1998. Proceedings. 1998 IEEE International Conference on, vol. 2. IEEE, 1998, pp. 11041109. 1

[9] K. Mombaur, M. Scheint, and M. Sobotka, "Optimal control and design of bipedal robots with compliance," atAutomatisierungstechnik, vol. 57, no. 7, pp. 349-359, 2009. 2

[10] K. Radkhah, D. Scholz, O. v. Stryk, M. Maus, and A. Seyfarth, "Towards human-like bipedal locomotion with threesegmented elastic legs," in Robotics (ISR), 2010 41st International Symposium on and 2010 6th German Conference on Robotics (ROBOTIK). VDE, 2010, pp. 1-8. 2

[11] M. Spong, "Modelling and control of robots with elastic joints," Journal Dynamic Systems, Measurement and Control, vol. 109, pp. 310-319, 1987. 2

[12] A. Wächter and L. T. Biegler, "On the implementation of an interior-point filter line-search algorithm for large-scale nonlinear programming," Mathematical Programming, vol. 106, pp. 25-57, 2006. 5

[13] P. R. Amestoy, I. S. Duff, J.-Y. L'Excellent, and J. Koster, "Mumps: a general purpose distributed memory sparse solver," in Applied Parallel Computing. New Paradigms for HPC in Industry and Academia. Springer, 2001, pp. 121-130. 5

[14] T. Moulard, F. Lamiraux, K. Bouyarmane, E. Yoshida, et al., "Roboptim: an optimization framework for robotics," in Robomec, 2013. 5 